\title{
NEW PERTURBATION BOUNDS FOR NONNEGATIVE AND POSITIVE POLAR FACTORS
}

\author{
HANYU Li, Hu YANG AND HUA SHAO
}

\begin{abstract}
The changes in the nonnegative and positive polar factors of generalized polar decomposition and polar decomposition are studied under the additive perturbation. Some new perturbation bounds are obtained. These bounds are different from precious ones in form and measure and, in some cases, may be smaller than the corresponding existing ones. Furthermore, the corresponding perturbation bounds for generalized nonnegative and positive polar factors of $(M, N)$ weighted polar decomposition are also presented as the straightforward corollaries.
\end{abstract}

Mathematics subject classification (2010): 15A09, 15A45, 65F35.

Keywords and phrases: polar decomposition, generalized polar decomposition, nonnegative polar factor, positive polar factor, perturbation bound, $(M, N)$ weighted polar decomposition.

\section{REFERENCES}

[1] A. BARRLUnd, Perturbation bounds on the polar decomposition, BIT. 30 (1989), 101-113.

[2] A. Ben-Israel and T. N. E. Greville, Generalized Inverses: Theory and Applications, 2nd ed., Springer, New York, 2003.

[3] R. Bhatia, Matrix Analysis, Springer-Verlag, New York, 1997.

[4] R. Bhatia, Modulus of continuity of the matrix absolute value, Indian J. Pure and Applied Math. 41 (2010), 99-111.

[5] X. S. CHEN, The absolute and relative perturbation bounds for the Hermitian positive semidefinite polar factor under unitarily invariant norm, J. South China Normal Univ. Natur. Sci. Ed. 3 (2010), 1-3 (in Chinese).

[6] X. S. Chen AND W. LI, Perturbation bounds on the polar decomposition under unitarily invariant norms, Math. Numer. Sin. 27 (2005), 121-128 (in Chinese).

[7] X. S. Chen AND W. LI, Relative perturbation bounds for subunitary polar factor under unitarily norm, Adv. Math. (China). 35 (2006), 178-184 (in Chinese).

[8] X. S. Chen And W. LI, Variations for $Q$ - and $H$-fators in the polar decomposition, Calcolo, 45 (2008), 99-109.

[9] X. S. Chen AND W. LI, W. SUn, Some new perturbation bounds for the generalized polar decomposition, BIT. 44 (2004), 237-244.

[10] C. H. Chen And J. G. Sun, Perturbation bounds for the polar factors, J. Comput. Math. 7 (1989), 397-401.

[11] C. DAVIS, W. M. KAnAN, The rotation of eigenvectors by a perturbation. III, SIAM J. Numer. Anal. 7 (1970), 1-46.

[12] N. J. Higham, Computing the polar decomposition-with applications, SIAM J. Sci. Statist. Comput. 7 (1986), 1160-1174.

[13] F. Kittaneh, Inequalities for the Schatten p-norm. III, Comm. Math. Phys. 104 (1986), 307-310.

[14] R. C. LI, A perturbation bound for the generalized polar decomposition, BIT. 33 (1993), 304-308.

[15] R. C. LI, New perturbation bounds for the unitary polar factor, SIAM J. Matrix Anal. Appl. 16 (1995), 327-332.

[16] R. C. LI, Relative perturbation theory: (II). Eigenspace and singular subspace variations, SIAM J. Matrix Anal. Appl. 20 (1998), 471-492. 
[17] R. C. LI, Relative perturbation bounds for positive polar factors of graded matrices, SIAM J. Matrix Anal. Appl. 27 (2006), 424-433.

[18] W. LI, Some new perturbation bounds for subunitary polar factors, Acta Math. Sin. 21 (2005), 15151520.

[19] W. LI, W. SUN, Perturbation bounds of unitary and subunitary polar factors, SIAM J. Matrix Anal. Appl. 23 (2002), 1183-1193.

[20] W. LI AND W. SUn, New perturbation bounds for unitary polar factors, SIAM J. Matrix Anal. Appl. 25 (2003), 362-372.

[21] W. Li AND W. Sun, Some remarks on the perturbation of polar decompositions for rectangular matrices, Numer. Linear Algebra Appl. 13 (2005), 327-338.

[22] R. Mathias, Perturbation bounds for the polar decomposition, SIAM J. Matrix Anal. Appl. 14 (1993), 588-593.

[23] C. R. Rao And S. K. Mitra, Generalized Inverses of Matrices and its Applications, Wiley, New York, 1971.

[24] C. R. Rao And M. B. Rao, Matrix Algebra and its Applications to Statistics and Econometrics, World Scientific, Hong Kong, 1998.

[25] J. G. Sun And C. H. Chen, Generalized polar decomposition, Math. Numer. Sin. 11 (1989), $262-$ 273 (in Chinese).

[26] C. F. VAN LOAN, Generalizing the singular value decomposition, SIAM J. Numer. Anal. 13 (1976), $76-83$.

[27] G. R. WANG, Y. M. WeI, AND S. Z. QIAO, Generalized Inverses: Theory and Computations, Science Press, Beijing, 2004.

[28] H. YANG AND H. Y. LI, Weighted polar decomposition and WGL partial ordering of rectangular complex matrices, SIAM J. Matrix Anal. Appl. 30 (2008), 898-924.

[29] H. YANG AND H. Y. LI, Weighted UDV*-decomposition and weighted spectral decomposition for rectangular matrices and their applications, Appl. Math. Comput. 198 (2008), 150-162.

[30] H. Yang AND H. Y. Li, Perturbation bounds for weighted polar decomposition in the weighted unitarily invariant norm, Numer. Linear Algebra Appl. 15 (2008), 685-700.

[31] H. YANG AND H. Y. LI, Weighted polar decomposition, J. Math. Res. Exposition. 29 (2009), 787-798. 